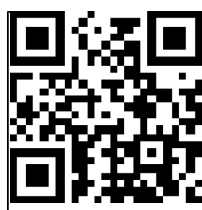

Editor's choice Scan to access more free content

10.1136/eb-2013-101634

${ }^{1}$ Department of Internal Medicine and Clinical Epidemiology, Princess Alexandra Hospital, Brisbane, Australia

${ }^{2}$ Department of Clinical Epidemiology and Biostatistics, McMaster University, Hamilton, Ontario, Canada

Correspondence to:

Dr lan A Scott

Department of Internal Medicine and Clinical Epidemiology,

Princess Alexandra Hospital, Brisbane, Queensland 4102, Australia;

ian.scott@health.qld.gov.au

\title{
Suggestions for improving guideline utility and trustworthiness
}

\section{Ian A Scott, ${ }^{1}$ Gordon H Guyatt ${ }^{2}$}

\section{Several underemphasised limitations of guidelines need proactive remediation in meeting the contemporary needs of clinicians}

Clinical practice guideline (CPG) panels are expected to abide by standards that ensure their processes are multidisciplinary, systematic and unbiased. ${ }^{1}$ Unfortunately, many CPGs fail to satisfy these standards. Only a third of 130 US guidelines produced by subspecialty societies between 2006 and 2011, ${ }^{2}$ satisfied more than 50\% of standards set by the Institute of Medicine (IOM-see table $1),{ }^{1}$ relating to panel composition, conflicts of interest, evidence synthesis, reconciliation of different interpretations of evidence and enumeration of treatment harms. Guidelines from other countries demonstrate similar deficiencies. ${ }^{3}$ Editorialists have identified the need for transparent measures of guideline trustworthiness, ${ }^{4}$ and some professional societies have issued rigorous standards for their guideline development panels. ${ }^{5}$ The fact that comparative studies have identified guidelines that more consistently meet most IOM standards ${ }^{6}{ }^{7}$ suggests that it is possible for more guideline panels to improve the quality and rigour of their processes.

In an era when clinicians are increasingly using CPGs to inform their care and guidelines are being increasingly used as reference standards for clinical audits, pay for performance schemes, public scorecards and medical litigation, guidelines must be both rigorously developed and mindful of challenges in implementing their recommendations. In this article, we explore problematic issues that have received limited attention to date in guideline appraisal tools and commentaries.

\section{Recommendations that conflict}

A medical defence organisation in Australia recently warned doctors that conflicting guideline recommendations around prostate cancer screening using prostaticspecific antigen (PSA) testing may render them individually liable to claims of delayed diagnosis. ${ }^{8}$ In this case, CPG issued from the Royal Australasian College of General Practitioners ${ }^{9}$ stated that men aged 55-69 years should not be offered PSA testing routinely whereas CPG from the Urological Society of Australia and New Zealand ${ }^{10}$ stated they should. Similar concerns about medicolegal risk arise from conflicting guideline recommendations pertaining to breast cancer screening. ${ }^{11}$ In both cases, discordance is most likely related to conflicts of interest and differing specialty perspectives of panelists. ${ }^{12}$

Such discordance is not unusual. Even within specialties, divided opinions often exist: US and European cardiologists hold differing views about anticoagulant use in acute coronary syndromes, ${ }^{13}$ while national and international specialty groups differ in their diagnostic and management approach to diabetes ${ }^{14}$ and hypertension. ${ }^{15}$
When recommendations conflict, clinicians would benefit from explicit statements regarding how guideline authors summarised and interpreted the evidence, and what values and preferences they adopted in trading off desirable and undesirable outcomes-the two key sources of differing guidance. As disagreements in evidence interpretation may be legitimate, and because values and preferences may well differ across jurisdictions or patient groups, differing recommendations may, at times, be appropriate. Nevertheless, reducing the frequency of conflicting recommendations is desirable, and fostering greater transparency in the formulation of recommendations is clearly desirable. These goals would be facilitated if guideline groups could, at a national and international level, harmonise their methodologies and collaborate in producing evidence summaries for common use. Such partnership ${ }^{1}$ could be fostered under the auspices of multinational agencies such as the WHO or the Guidelines International Network. ${ }^{16}$

Advantages of such collaboration would arise not only from a greater likelihood of consistent interpretation, but also in efficiency: to have multiple groups searching the same literature and conducting timeconsuming evaluation of that literature is hugely inefficient. Collaboration may also allow panels to review and, if satisfied, endorse each other's recommendations, ${ }^{17}$ thus preventing further needless duplication in guideline development. At the same time, 'globalising the evidence while localising the recommendations ${ }^{18}$ recognises the need to customise guidelines according to values and preferences of target populations or regional resource constraints or circumstances. Few guidelines will be applicable across countries of very different wealth. In the absence of demonstrable variation in characteristics and preferences of populations within a country, national borders may be the reasonable default localising boundary.

\section{Recommendations that go beyond robust evidence}

Examples exist of major guideline recommendations having to be substantively revised, even reversed, in response to an enlarging totality of evidence. In some instances, guideline panels, in formulating their original recommendations, may have been prematurely swayed by dramatic results reported in single randomised trials, many involving small samples and/or stopped early for apparent benefit. ${ }^{19}$ Such enthusiasm, possibly fanned in some instances by conflicts of interest and undue influence of industry, turned out to be misplaced when larger subsequent trials failed either to reproduce such effects or disclosed serious adverse outcomes. Examples include $\beta$-blockers as cardioprotective agents in patients undergoing non-cardiac surgery, intensive insulin therapy in critically ill patients and activated protein $\mathrm{C}$ infusions in 
Table 1 Institute of Medicine standards for developing trustworthy clinical practice guidelines ${ }^{1}$

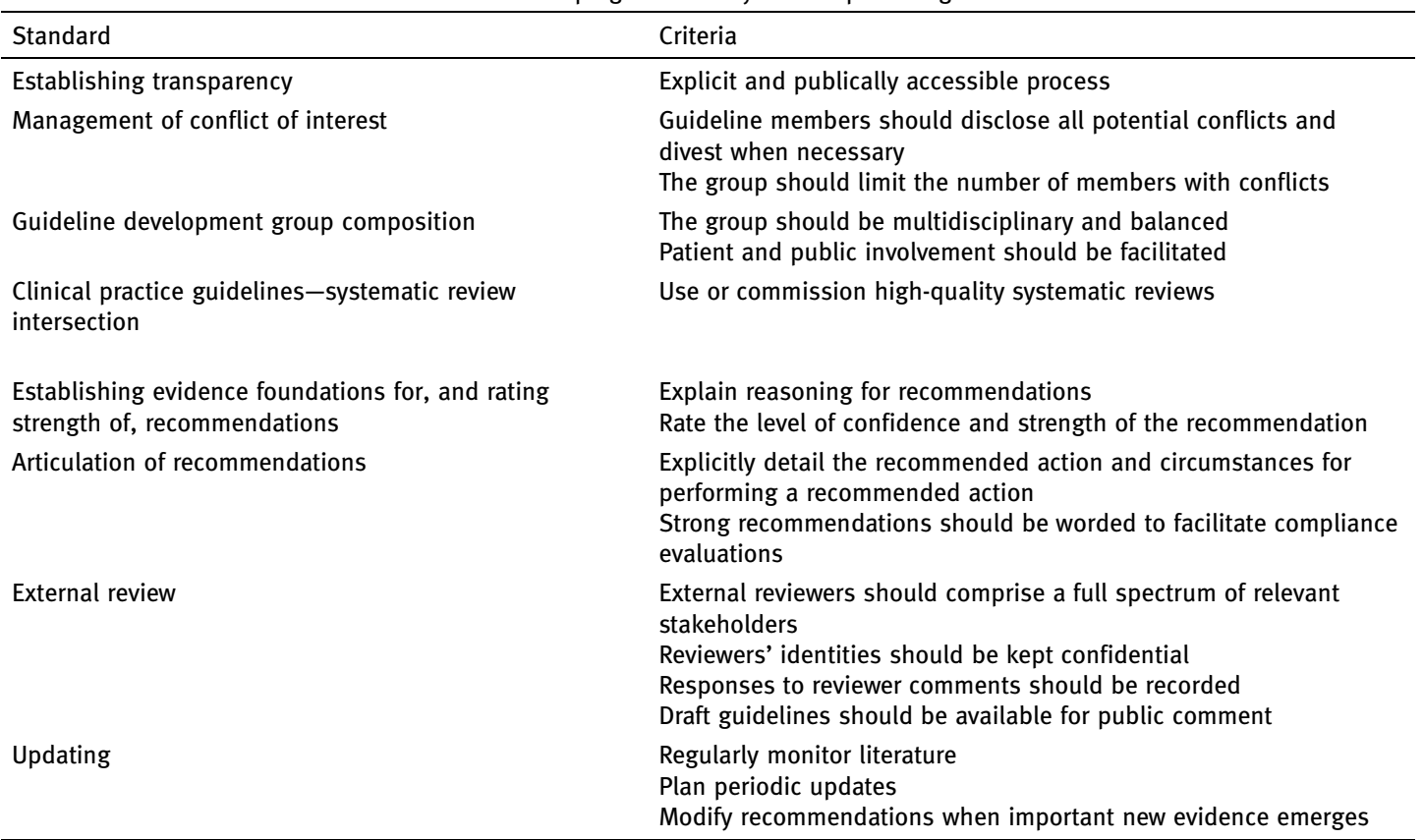

patients with septic shock. ${ }^{19}$ In other instances, guidelines have advocated increasingly tight control of blood glucose and blood pressure, often relying on logical inference, to extend thresholds beyond the available evidence, with later trials showing no benefits and increased hazards. ${ }^{20}{ }^{21}$ Guideline panels need to be appropriately critical about existing evidence, and offer correspondingly conservative recommendations.

\section{Recommendations that lag behind robust evidence}

Guideline panels must establish procedures for regularly and systematically reviewing the evidence base and updating their recommendations in a timely manner in response to new compelling evidence. This helps prevent recently released guidelines simply ignoring such evidence, or outdated guidelines persisting long after the publication of new research.

As an example, nephrology guidelines released in 2006 and financially supported by manufacturers of erythropoiten recommended a target haemoglobin $(\mathrm{Hb})$ level between 110 and $130 \mathrm{mmol} / \mathrm{L}$ in patients with endstage renal disease undergoing dialysis. ${ }^{22}$ This recommendation flew in the face of trials published over the previous 5 years showing that $\mathrm{Hb}$ levels above $120 \mathrm{mmol} / \mathrm{L}$ were associated with increased risk of cardiovascular events and death. ${ }^{23}$

US guidelines addressing stable ischaemic heart disease published in $2002^{24}$ and not updated until $2012^{25}$ present another example of failure to keep up to date. During the hiatus, clinicians faced recommendations that patients with stable angina and non-critical coronary artery disease be offered coronary revascularisation in addition to optimal medical therapy, despite the publication of large trials showing that optimal medical management and lifestyle modifications achieved similar outcomes of symptom control, morbidity and mortality. ${ }^{25}$

\section{Recommendations based on low-quality evidence}

Guideline panels frequently confront topics for which high-quality evidence is unavailable-either because efforts to gather evidence were inadequate or because such evidence simply does not exist.

In regard to the former, systematic searching methods have evolved to ensure that all relevant published studies are available to authors of systematic reviews and, via them, guideline panels. Such methods are, however, only helpful when they are used. A recent review of Australian CPGs found that only 16\% of them used systematic literature reviews as a basis for recommendations, ${ }^{26}$ running a risk of guidelines based on unrepresentative evidence. $^{27}$

With regard to the latter, recommendations have to be based on low or very low quality evidence if this is the best systematic reviews can uncover. Low-quality evidence-which runs a greater risk of subsequent reversal than evidence warranting greater confidence-underpins $85 \%$ of major CPG recommendations in cardiovascular medicine ${ }^{28}$ and more than $50 \%$ in infectious disease medicine. ${ }^{29}$

Accordingly, recommendations based on low-quality evidence should rarely be used to generate quality metrics or legitimise established beliefs and practice styles. For instance, guidelines for systolic heart failure (SHF) have, for many years, recommended low salt diets in patients with moderate-to-severe $\mathrm{SHF}^{30} \mathrm{~A}$ recent meta-analysis of randomised trials suggests, however, that such diets increase mortality. ${ }^{31}$ Previous intensive care guidelines for severe sepsis recommended use of either colloid solutions (starch or albumen) or crystalloids to treat sepsis-induced hypovolaemia ${ }^{32}$ and insulin therapy to achieve close to normoglycaemic blood sugar levels. ${ }^{33}$ Subsequent high-quality trials have revealed increased risk of death and need for renal replacement therapy from commonly used starch solutions, ${ }^{34}{ }^{35}$ and 
raised the possibility of increased mortality from aggressive blood sugar control. ${ }^{36}$ A final example: a recent adequately powered trial showed no reduction in mortality from the use of intra-aortic balloons in cases of cardiogenic shock following acute myocardial infarction, ${ }^{37}$ despite previous recommendations ${ }^{38}$ based on observational data suggesting benefit in this highly lethal condition.

Guideline recommendations should always be accompanied by a systematically derived summary of best available evidence that rates evidence quality and links it with the strength of recommendations, ideally using the GRADE system. ${ }^{39}$ Where evidence quality is low, weak recommendations should mostly apply. The need to subject expert opinion to explicit, transparent consensus methods that minimise domination of opinion by one or a few panelists becomes even more paramount when evidence is low quality. ${ }^{40}$ Where panelists identify recommendations for which they fail to reach consensus, they need to state the reasons why. In the rare situation in which evidence is of such low quality that, even after careful consideration of all factors that may bear on decision making, ${ }^{41}$ panelists regard any recommendation as being too speculative, they may refrain from making a recommendation, clearly stating the reasons why. Doing so however means that the potentially puzzled clinician is left without guidance.

Strong recommendations based on low-quality evidence carry the risks of encouraging uniform practice that may not be in patients' best interest and inhibiting research that could clarify the magnitude of benefit and harm. In general, panelists should be very cautious in issuing strong recommendations (ie, those that apply to all or almost all patients) in the face of low-quality evidence-if one is not sure of the effects, it is difficult to be sure of the right course of action. Although strong recommendations may be justified in certain situations of low quality of evidence (see table 2 ), ${ }^{41}$ guideline users should, in general, regard strong recommendations based on low quality evidence with circumspection.

\section{Recommendations that do not reconcile individualised estimates of benefits and harms}

Despite poor reporting of care-related harm in many clinical trials, guideline authors should, as far as possible, express the benefit-harm trade-offs in terms of absolute risk of patient-important events. Recommendations for universal prophylaxis for venous thromboembolism (VTE) in hospitalised general medical patients overlook the fact that symptomatic VTE occurs in only 2/1000 untreated patients without risk factors, that prophylaxis has no impact on mortality, and that for every 1000 average-risk patients treated with prophylactic heparin, three episodes of pulmonary embolism are prevented at the cost of four major bleeding episodes. ${ }^{43}$

Different guidelines also vary in the levels of absolute disease risk at which they recommend initiation of preventive treatments such as statins. ${ }^{44}$ In other instances, treatment is recommended solely on the basis of single risk factors-such as cholesterol or blood pressure levels-exceeding certain threshold values, in the absence of a multifactorial estimate of patients' overall disease risk. ${ }^{45}$ Both approaches discourage prioritisation

Table 2 Situations for which currently available low-quality evidence is associated with strong recommendations that could change as new high-quality evidence emerges*

\begin{abstract}
(1) When low-quality evidence suggests benefit in a life-threatening situation (evidence regarding harms can be low or high)
\end{abstract}

(2) When low-quality evidence suggests benefit and high-quality evidence suggests harm or a very high cost

(3) When low-quality evidence suggests benefit equivalence of two alternatives, but high-quality evidence of less harm for one of the competing alternatives

(4) When high-quality evidence suggests benefit equivalence of two alternatives and low-quality evidence suggests harm in one alternative

(5) When high-quality evidence suggests modest benefits catastrophic harm and low/very low-quality evidence suggests possibility of

Fresh frozen plasma or vitamin $\mathrm{K}$ in a patient receiving warfarin with elevated INR and an intracranial bleed. Only low-quality evidence supports the benefits of limiting the extent of the bleeding

Head-to-toe CT/MRI screening for cancer. Low-quality evidence of benefit of early detection but high-quality evidence of possible harm and/or high cost (strong recommendation against this strategy)

Helicobacter pylori eradication in patients with early stage gastric MALT lymphoma with $H$ pylori positive. Low-quality evidence suggests that initial $H$ pylori eradication results in similar rates of complete response in comparison with the alternatives of radiation therapy or gastrectomy; high-quality evidence suggests less harm/morbidity from $\mathrm{H}$ pylori eradication

Hypertension in women planning conception and in pregnancy. Strong recommendations for labetalol and nifedipine and strong recommendations against ACE inhibitors and angiotensin receptor blockers (ARB). All agents have high-quality evidence of equivalent beneficial outcomes, with low-quality evidence for greater adverse effects with ACE inhibitors and ARBs

Testosterone in males with or at risk of prostate cancer. High-quality evidence for moderate benefits of testosterone treatment in men with symptomatic androgen deficiency to improve bone mineral density and muscle strength. Low-quality evidence for harm in patients with or at high risk of prostate cancer

*Adapted from reference ${ }^{42}$.

INR, international normalised ratio; MALT, mucosa-associated lymphoid tissue. 
of treatment for patients at higher absolute risk which could potentially prevent more adverse events and at a lower cost within a given population. ${ }^{45}$ As a final note, recommendations for add-on incremental therapy that target multiple risk factors in the same individual may be inadvisable when very small benefits do not warrant exposure to associated harms and burdens. ${ }^{46}$

\section{Recommendations that focus on single diseases and ignore comorbidities}

Most patients with a chronic disease have multiple comorbidities that single-disease guidelines do not address. Most CPGs do not consider competing risks ${ }^{47}$ and rarely consider applicability to individuals with limited life expectancy who are unlikely to benefit from long-term preventive treatments. ${ }^{48}$ Hardly ever do they address when to stop one or more of multiple chronic treatments, despite high-quality evidence that define circumstances where antihypertensive, hypoglycaemic and psychotropic medications can be safely discontinued. ${ }^{49}$

Several strategies may render CPGs more useful in the context of multimorbidity: (1) cross-referencing guidelines dealing with other complaints commonly associated with the index condition (eg, depression, pain, cognitive impairment and falls in patients with heart failure, most of whom are elderly $)^{50}$; (2) emphasising benefits (or harms) of disease-specific treatments with regard to other commonly co-occurring diseases that may be 'concordant' (diseases such as diabetes, hypertension or coronary artery disease which share a common management plan) or 'discordant' (diseases such as diabetes, asthma and depression in which management plans differ and may interact ${ }^{51}$ ); (3) estimating the time at which slowly accruing treatment-related benefits outweigh immediate or constant rate harms, and if this time exceeds expected lifespan, recommending that treatments be discontinued or not initiated; ${ }^{52}$ and (4) liberalising and customising treatment targets (such as the desired levels of glucose and blood pressure control) according to age and homoeostatic reserve. ${ }^{53}$

\section{Recommendations that are insensitive to patient preferences}

Patients may value outcomes differently from guideline authors, including critically important outcomes such as death or serious morbid events. ${ }^{54}$ Many patients place as much, if not more, emphasis on avoiding treatment-related short-term toxicity, even if uncommon, than on primary effects in lowering future disease risk. ${ }^{55}$ Ideally, guideline developers should systematically review available evidence regarding risk perceptions and care preferences of their target populations and develop recommendations accordingly. Antithrombotic guidelines from the American College of Chest Physicians demonstrate this approach. ${ }^{56}$ Guideline panels should state explicitly the value and preferences structure underlying their recommendations, with statements attached to recommendations in which patient preferences are likely to be particularly salient to decision-making.

Guideline developers should promote informing and empowering patients to share in decision making. Strategies include guideline chapters that encourage clinicians to adopt a shared decision making approach, patient versions of guidelines, risk communication tools (graphs and pictograms), values clarification tools and complete patient decision aids and, perhaps most usefully, tools for use during time constrained patient-physician interactions tied to specific recommendations. ${ }^{57}$

\section{Recommendations that ignore implementation challenges}

Limited resources, organisational and cultural barriers, and clinicians lacking skills to optimally implement recommendations constitute barriers to guideline uptake. $^{58}$ Although guideline developers cannot deal with every contingency, they should ideally survey a representative sample of end-users, identify likely roadblocks to implementation and proffer potential solutions.

Resource implications of guideline recommendations, likely to be an increasing challenge to implementation in fiscally tight healthcare systems, were not explicitly considered in almost half of 30 US specialty society guidelines, and when they were, only half consistently used a formal method. ${ }^{59}$ Although no universally agreed method for incorporating economic analyses into CPGs currently exists, guideline panels should ideally consider cost-effectiveness when determining the direction and strength of their recommendations. ${ }^{60}$ They should also revise their recommendations if needed in response to formal economic evaluations of adherence to guideline recommendations and costs of implementation strategies in target populations. ${ }^{61}$

\section{Recommendations that are poorly responsive to a changing environment}

Once CPGs are released, guideline panels should adopt procedures that allow recommendations and their methods of implementation to be revised, in a timely fashion, in response to user feedback and evaluations of guideline impact from qualitative research and clinical audit. $^{62}$ Guidelines need to become living documents capable of rapid updating as important new evidence or suggestions for improving content and format emerge. Recently released cancer CPGs from the Cancer Council of Australia use an online 'wiki' format to allow readers -whether they are patients, carers or clinicians-to submit suggestions that a working group then considers. $^{63}$

\section{Are we asking too much of guideline panels?}

Guideline panels may feel that they have enough with which to contend without imposing the additional requests discussed in this article. They may bemoan the cost and effort of complying with onerous standards. However, the issues discussed above encompass the challenges that clinicians have to grapple with in everyday practice while caring for individual patients and for which clinicians look to clinical guidelines for assistance. Fortunately, various electronic support systems are being developed that may greatly assist panels in retrieving evidence ${ }^{64}$ and authoring actionable guidelines. ${ }^{65}$ Considerable time and resources are currently expended worldwide on guideline development. Fewer 
but better resourced and rigorous guideline panels may be able to implement the entire range of strategies that maximise guideline trustworthiness.

Contributors IAS conceived the ideas, gathered data and wrote the draft manuscript; GHG assisted in gathering data, critically appraised the manuscript, and revised the text. Data were obtained from published research kept on file by IAS and supplemented by additional references supplied by GHG. IAS is a general physician and clinical epidemiologist with long-standing interest in guideline development and dissemination, has coauthored cardiology guidelines, and has published research articles pertaining to guideline methodology. GHG is a general internist and clinical epidemiologist who has published extensively on guideline methods, developed with others the GRADE system of grading recommendations and assessing development of evidence for clinical guidelines, and is a lead methodologist for the American College of Chest Physicians guidelines on antithrombotic treatment.

\section{Competing interests None.}

\section{References}

1. Graham R, Mancher M, Wolman DM, et al. eds. Clinical practice guidelines we can trust. Washington, DC: National Academies Press, 2011.

2. Kung J, Miller RR, Mackowiak PA. Failure of clinical practice guidelines to meet Institute of Medicine standards: two more decades of little, if any, progress. Arch Intern Med 2012;172:1628-33.

3. Alonso-Coello P, Irfan A, Solà I, et al. The quality of clinical practice guidelines over the last two decades: a systematic review of guideline appraisal studies. Qual Saf Health Care 2010;19:e58.

4. Ransohoff DF, Pignone M, Soz HC. How to decide whether a clinical practice guideline is trustworthy. JAMA 2013;309:139-40.

5. Jacobs AK, Kushner FG, Ettinger SM, et al. ACCF/AHA Clinical Practice Guideline Methodology Summit Report: a report of the American College of Cardiology Foundation/American Heart Association Task Force on practice guidelines. Circulation 2013;127:268-310.

6. Fervers B, Burgers JS, Haugh MC, et al. Predictors of high quality clinical practice guidelines: examples in oncology. Int $J$ Qual Health Care 2005;17:123-32.

7. Bennett WL, Odelola OA, Wilson LM, et al. Evaluation of guideline recommendations on oral medications for type 2 diabetes mellitus. A systematic review. Ann Intern Med 2012;156:27-36.

8. Bradley A. Doctors sued amid PSA confusion. Conflicting guidelines spark MDO warning. Australian Doctor. July 6 2012:1.

9. Royal Australasian College of General Practitioners. Guidelines for preventive activities in general practice (the Red Book). 8th edn. http://www.racgp.org.au/download/Documents/Guidelines/ Redbook8/redbook8.pdf (accessed 15 Nov 2012).

10. Urological Society of Australia and New Zealand PSA Testing Policy 2009. http://www.usanz.org.au/uploads/29168/ufiles/ USANZ_2009_PSA_Testing_Policy_Final1.pdf (accessed 15 Nov 2012).

11. Kachalia A, Mello MM. Breast cancer screening. Conflicting guidelines and medicolegal risk. JAMA 2013;309:2555-6.

12. Norris SL, Burda BU, Holmer HK, et al. Author's specialty and conflicts of interest contribute to conflicting guidelines for screening mammography. J Clin Epidemiol 2012;65:725-33.
13. Eikelboom JW, Guyatt G, Hirsh JW. Guidelines for anticoagulant use in acute coronary syndromes. Lancet 2008;371:1559-61.

14. Kahn R, Gale EAM. Gridlocked guidelines for diabetes. Lancet 2010;375:2203-4.

15. McAlister FA, Campbell NRC, Zarnke K, et al. The management of hypertension in Canada: a review of current guidelines, their shortcomings and implications for the future. CMAJ 2001;164:517-22.

16. Qaseem A, Forland F, Macbeth F, et al. Guidelines International Network: toward international standards for clinical practice guidelines. Ann Intern Med 2012;156:525-31.

17. Basch EM, Somerfield MR, Beer TM, et al. American Society of Clinical Oncology endorsement of the Cancer Care Ontario practice guideline on nonhormonal therapy for men with metastatic hormone-refractory (castration-resistant) prostate cancer. J Clin Oncol 2007;25:5313-18.

18. Eisenberg JM. Globalize the evidence, localize the decision: evidence-based medicine and international diversity. Health Aff 2002;21:166-8.

19. Guyatt GH, Briel M, Glasziou P, et al. Problems of stopping trials early. BMJ 2012;344:e3863.

20. American Diabetes Association. Standards of medical care in diabetes: 2010. Diabetes Care 2010;33(Suppl 1):S11-61.

21. Chobanian AV, Bakris GL, Black HR, et al. National Heart, Lung, and Blood Institute Joint National Committee on Prevention, Detection, Evaluation, and Treatment of High Blood Pressure. The seventh report of the Joint National Committee on Prevention, Detection, Evaluation, and Treatment of High Blood Pressure; the JNC 7 report. JAMA 2003;289:2560-72.

22. No authors listed. II. Clinical practice guidelines and clinical practice recommendations for anaemia in chronic kidney disease in adults. Am J Kidney Dis 2006;47:S16-85.

23. Coyne DW. Influence of industry on renal guideline development. Clin J Am Soc Nephrol 2007;2:3-7.

24. Gibbons RJ, Abrams J, Chatterjee K, et al. American College of Cardiology. ACC/AHA 2002 guideline update for the management of patients with chronic stable angina-summary article: a report of the American College of Cardiology/ American Heart Association Task Force on practice guidelines (Committee on the Management of Patients with Chronic Stable Angina). J Am Coll Cardiol 2003;41:159-68.

25. Qaseem A, Fihn SD, Dallas P, et al. for the Clinical Guidelines Committee of the American College of Physicians. Management of stable ischemic heart disease: summary of a Clinical Practice Guideline from the American College of Physicians/American College of Cardiology/Foundation/American Heart Association/ American Association for Thoracic Surgery/Preventive Cardiovascular Nurses Association/Society of Thoracic Surgeons. Ann Intern Med 2012;157:735-43.

26. Buchan HA, Currie KC, Lourey EJ, et al. Australian clinical practice guidelines-a national study. Med J Aust 2010;192:490-4.

27. Hoffmann T, Erueti C, Thorning S, et al. The scatter of research: cross sectional comparison of randomised trials and systematic reviews across specialties. BMJ 2012;344:e3223.

28. Tricoci P, Allen JM, Kramer JM, et al. Scientific evidence underlying the ACC/AHA clinical practice guidelines. JAMA 2009;301:831-41.

29. Lee DH, Vielemeyer O. Analysis of overall level of evidence behind Infectious Diseases Society of America practice guidelines. Arch Intern Med 2011;171:18-22.

30. Heart Failure Society of America. HFSA 2006 comprehensive heart failure practice guidelines. J Card Fail 2006;12:e1-2.

31. DiNicolantonio JJ, Di Pasquale P, Taylor RS, et al. Low sodium versus normal sodium diets in systolic heart failure: systematic review and meta-analysis. Heart Published Online First: 12 Mar 2013. 
32. Dellinger RP, Levy MM, Carlet JM, et al. Surviving sepsis campaign: international guidelines for management of severe sepsis and septic shock: 2008. Intensive Care Med 2008;34:17-60.

33. American Diabetes Association. American College of Endocrinology and American Diabetes Association Consensus statement on inpatient diabetes and glycemic control: a call to action. Diabetes Care 2006;29:1955-62.

34. Brunkhorst FM, Engel C, Bloos F, et al. Intensive insulin therapy and pentastarch resuscitation in severe sepsis. $N$ Engl J Med 2008;358:125-39.

35. Perner A, Haase N, Guttormsen AB, et al. Hydroxyethyl starch 130/0.42 versus Ringer's acetate in severe sepsis. $N$ Engl J Med 2012;367:124-34.

36. The NICE-SUGAR Study Investigators. Intensive versus conventional glucose control in critically ill patients. $N$ Engl $J$ Med 2009;360:1283-97.

37. Thiele H, Zeymer U, Neumann FJ, et al. Intraaortic balloon support for myocardial infarction with cardiogenic shock. N Engl J Med 2012;367:1287-96.

38. Van de Werf F, Bax J, Betriu A, et al. Management of acute myocardial infarction in patients with persistent ST-segment elevation: the task force on the management of ST-segment elevation acute myocardial infarction of the European Society of Cardiology. Eur Heart J 2008;29:2909-45.

39. Guyatt GH, Oxman AD, Vist GE, et al. GRADE: an emerging consensus on rating quality of evidence and strength of recommendations. BMJ 2008;336:924-6.

40. Loblaw DA, Prestrud AA, Somerfield MR, et al. American Society of Clinical Oncology Clinical Practice Guidelines: formal systematic review-based consensus methodology. J Clin Oncol 2012;30:3136-40.

41. Petitti DB, Teutsch SM, Barton MB, et al. Update on the methods of the U.S. Preventive Services Task Force: insufficient evidence. Ann Intern Med 2009;150:199-205.

42. Andrews JC, Schunemann HG, Oxman AD, et al. GRADE guidelines 15: going from evidence to recommendation: determinants of a recommendation's direction and strength. J Clin Epidemiol 2013;66:726-35.

43. Lederle FA, Zylla D, Macdonald R, et al. Venous thromboembolism prophylaxis in hospitalized medical patients and those with stroke: a background review for an American College of Physicians clinical practice guideline. Ann Intern Med 2011;155:602-15.

44. Manuel DG, Kwong K, Tanuseputro P, et al. Effectiveness and efficiency of different guidelines on statin treatment for preventing deaths from coronary heart disease: modelling study. BMJ 2006;332:1419-23.

45. Eddy DM, Adler J, Patterson B, et al. Individualised guidelines: the potential for increasing quality and reducing costs. Ann Intern Med 2011;154:627-34.

46. Avins AL. When clinical practice guidelines meet the black box. Arch Intern Med 2010;170:1013-14.

47. Vitry AI, Zhang Y. Quality of Australian clinical guidelines and relevance to care of older people with multiple comorbid conditions. Med J Aust 2008;189:360-5.

48. Braithwaite RS. Can life expectancy and QALYs be improved by a framework for deciding whether to apply clinical guidelines to patients with severe comorbid disease? Med Decis Making 2011;31:582-95.
49. Scott IA, Gray LA, Martin JH, et al. Deciding when to stop: towards evidence-based deprescribing of drugs in older populations. Evid Based Med 2012;18:121-4.

50. Guthrie B, Payne K, Alderson P, et al. Adapting clinical guidelines to take account of multimorbidity. BMJ 2012;345: e6341.

51. Piette JD, Kerr EA. The impact of comorbid chronic conditions on diabetes care. Diabetes Care 2006;29:725-31.

52. Braithwaite RS, Fiellin D, Justice AC. The payoff time: a flexible framework to help clinicians decide when patients with comorbid disease are not likely to benefit from practice guidelines. Med Care 2009;47:610-17.

53. Alexander KP, Newby K, Cannon CP, et al. Acute coronary care in the elderly, Part I. Non-ST-segment-elevation acute coronary syndromes. A scientific statement for healthcare professionals from the American Heart Association Council on Clinical Cardiology in collaboration with the Society of Geriatric Cardiology. Circulation 2007;115:2549-69.

54. Protheroe J, Fahey T, Montgomery AA, et al. The impact of patients' preferences on the treatment of atrial fibrillation: observational study of patient based decision analysis. $B M J$ 2000;320:1380-4.

55. Fried TR, Tinetti ME, Towle V, et al. Effects of benefits and harms on older persons' willingness to take medication for primary cardiovascular prevention. Arch Intern Med 2011;171:923-8.

56. MacLean S, Mulla S, Akl EA, et al. Patient values and preferences in decision making for antithrombotic therapy: a systematic review: antithrombotic therapy and prevention of thrombosis, 9th ed: American College of Chest Physicians Evidence-Based Clinical Practice Guidelines. Chest 2012;141 (Suppl 2):e1S-23S.

57. van der Weijden T, Pieterse AH, Koelewijn-van Loon MS, et al. How can clinical practice guidelines be adapted to facilitate shared decision making? A qualitative key-informant study. BMJ Qual Saf 2013;22:855-63.

58. Cabana MD, Rand CS, Powe NR, et al. Why don't physicians follow clinical practice guidelines? A framework for improvement. JAMA 1999;282:1458-65.

59. Schwartz JAT, Pearson SD. Cost consideration in the clinical guidance documents of physician specialty societies in the United States. JAMA Intern Med 2013;173:1091-7.

60. Guyatt GH, Oxman AD, Kunz R, et al. GRADE: Incorporating considerations of resources use into grading recommendations. BMJ 2008;336:1170-3.

61. Hoomans T, Ament AJHA, Evers SA, et al. Implementing guidelines into clinical practice: what is the value? J Evalu Clin Pract 2011;17:606-14.

62. Zuiderent-Jerak T, Forland F, Macbeth F. Guidelines should reflect all knowledge, not just clinical trials. BMJ 2012;345:e6702.

63. Clinical Guidelines Network Cancer Council Australia Development of Clinical Practice Guidelines using Cancer Council Australia's Cancer Guidelines Wiki. Handbook 2012. http://wiki.cancer.org.au (accessed 11 Jan 2013).

64. Tsafnat G, Dunn A, Glasziou P, et al. The automation of systematic reviews. BMJ 2013;346:f139.

65. Vandvik PO, Brandt L, Alonso-Coello P, et al. Creating clinical practice guidelines we can trust, use and share: a new era is imminent. Chest 2013;144:381-9. 